\title{
Carta al Editor \\ Es erróneo hacer una asociación directa entre la detección de Helicobacter spp. y gastritis en caninos
}

\section{Letter to the editor: \\ It is erroneous to directly associate the detection of Helicobacter spp. with gastritis in canines}

\author{
Marcela Suárez-Esquivel ${ }^{1,}$ Elías Barquero-Calvo ${ }^{1 凶}$
}

1 Programa de Investigación en Enfermedades Tropicales, Escuela de Medicina Veterinaria, Universidad Nacional. marcela.suarez.esquivel@una.cr

Palabras clave: Helicobacter, gastritis, carga bacteriana, Helicobacter pylori.

Estimado editor,

Tal y como sucede en seres humanos, en los perros, se ha propuesto que la presencia de Helicobacter es importante en la generación de gastritis y cáncer (Jergens et al. 2009). No obstante, a pesar de diversos esfuerzos independientes, aún existe controversia respecto a la verdadera capacidad de las distintas especies Helicobacter para causar lesiones gástricas en estos animales. Se debe, en parte, a que la gastritis es una enfermedad multifactorial, en la cual, influyen diversos elementos, por ejemplo: la presencia de enfermedades sistémicas, el tipo de dieta, las influencias ambientales, el estrés y otros factores de comportamiento (Jergens et al. 2009).

La mayoría de los reportes, que implican a Helicobacter como causa de lesiones gástricas en animales, se generó a partir de la utilización de ratones o gerbos infectados de forma experimental (De Bock et al. 2006; Joosten et al. 2013). Los estudios enfocados en investigar infecciones adquiridas de forma natural, han sido incapaces de establecer una correlación entre la colonización por Helicobacter y la presencia de enfermedad (Harbour \& Sutton 2008). Recientemente, se publicó un artículo en el cual se demuestra que el $95 \%$ de los perros, sometidos a observación (80 en total), estaban colonizados con Helicobacter spp. Lo cual indica que esta bacteria es sumamente común en la microbiota gástrica de los perros (Suárez-Esquivel et al. 2017). Así como se demuestra en estudios de otros autores, en este trabajo tampoco se encontró una asociación entre la presencia de lesiones gastroscópicas o histopatológicas con la presencia o la cantidad de Helicobacter spp. detectada en la mucosa gástrica (Suárez-Esquivel et al. 2017). Además, tampoco se detecta muestra positiva alguna para la especie $H$. pylori; lo cual, sugiere que los perros no son portadores importantes de esta especie. Por tanto, no representan un riesgo zoonótico significativo para esta especie en particular.

Además, la gastritis es una enfermedad multifactorial. También, existen otras causas que dificultan establecer una asociación clara entra la presencia de las distintas especies de Helicobacter y gastritis:

\ Autor encargado de la correspondencia: elias.barquero.calvo@una.cr 
1. Actualmente, la taxonomía del género Helicobacter es confusa. Así lo demostraron, recientemente, Joosten et al. (2016) a través de la secuenciación de genomas completos. Algunas cepas virulentas de $H$. heilmannii no se consideran $H$. heilmanii, sino una nueva especie propuesta: $H$. ailurogastricus. La mayoría de la filogenia actual se basa en el análisis del gen $16 \mathrm{~S}$ rRNA, lo cual no permite diferenciar las cepas estrechamente relacionadas porque las secuencias son muy similares. Esta polémica ha permitido establecer un número aproximado de 10 Candidatus y/o especies no validadas (Mitchell et al. 2014). Es probable que se requiera de la secuenciación completa de más genomas para poseer, con claridad, una clasificación a nivel de especie en el género Helicobacter.

2. Los factores de virulencia de las especies de Helicobacter, que colonizan los perros, aún no se conocen con certeza. Se ha descrito que hay variaciones en el grado de virulencia en perros infectados experimentalmente con bacterias de la especie $H$. heilmannii; sin embargo, aún no se ha podido establecer la causa de estas diferencias (Joosten et al. 2013). Una diferencia importante consiste en que los genes $\operatorname{cag} A$ y $v a c A$, descritos previamente en $H$. pylori, que determinan en gran medida la virulencia de esta especie, no se encuentran en las especies caninas. Se ha reportado, además, cómo algunas cepas de H. suis muestran distintas capacidades de colonización; no obstante, los autores lo atribuyen más a particularidades del hospedero que a características de la bacteria (Flahou et al. 2012).

3. Las infecciones, causadas por varias especies de Helicobacter de forma simultánea, han sido reportadas previamente, las cuales parecen ser muy frecuentes (Amorim et al. 2015). Estos datos complican, no sólo a nivel de diagnóstico, determinar las especies presentes en los animales, sino que afecta establecer, con certeza, si alguna de las especies presentes podría tener algún efecto en el desarrollo de lesiones gástricas.

El conocimiento actual, en términos de taxonomía y patogénesis de las especies de Helicobacter que colonizan los caninos, es insuficiente para diferenciar con certeza entre cepas potencialmente patógenas y no patógenas. En consecuencia, la detección de Helicobacter, aún en altas cantidades, no se debe asociar de forma directa como causa de gastritis, sin antes considerar los múltiples factores que podrían originar esta patología.

\begin{abstract}
It has been proposed that Helicobacter may play a significant role in the generation of gastritis and cancer in dogs. However, despite several independent efforts, there is still controversy regarding the true ability of Helicobacter to cause gastric lesions in these animals. The foregoing is due in part to the fact that gastritis is a multifactorial disease. Studies focused on naturally acquired infections have been unable to establish a positive correlation between Helicobacter colonization and gastritis. Establishing a clear association between the presence of Helicobacter and gastritis may be difficult due to the following reasons: (i) taxonomy of the genus Helicobacter remains unclear, (ii) virulence factors of Helicobacter species that colonize dogs are not well known, and (iii) simultaneous infections have been reported with several species of Helicobacter. Current knowledge in taxonomy and pathogenesis of Helicobacter species is insufficient to differentiate between pathogenic and non-pathogenic strains. Therefore, no direct association should be made between the detection of Helicobacter and gastritis.
\end{abstract}

Key words: Helicobacter, gastritis, bacterial load, Helicobacter pylori 


\section{Referencias}

Amorim, I., Smet, A., Alves, O., Teixeira, S., Saraiva, A.L., Taulescu, M., Reis, C., Haesebrouck, F. \& Gärtner, F. 2015. Presence and significance of Helicobacter spp. in the gastric mucosa of Portuguese dogs. Gut Pathog. 7: 12. doi:10.1186/s13099-015-0057-1

De Bock, M., Decostere, A., Hellemans, A., Haesebrouck, F. \& Ducatelle, R. 2006. Helicobacter felis and Helicobacter bizzozeronii induce gastric parietal cell loss in Mongolian gerbils. Microbes Infect. 8 (2): 503-510. doi:10.1016/j.micinf.2005.08.003

Flahou, B., Van Deun, K., Pasmans, F., Smet, A., Volf, J., Rychlik, I., Ducatelle, R. \& Haesebrouck, F. 2012. The local immune response of mice after Helicobacter suis infection: strain differences and distinction with Helicobacter pylori. Vet. Res. 43: 75. doi:10.1186/1297-9716-43-75

Harbour, S. \& Sutton, P. 2008. Immunogenicity and pathogenicity of Helicobacter infections of veterinary animals. Vet. Immunol. Immunopathol. 122 (3-4): 191-203. doi:10.1016/j.vetimm.2007.12.003

Jergens, A.E., Pressel, M., Crandell, J., Morrison, J.A., Sorden, S.D., Haynes, J., Craven, M., Baumgart, M. \& Simpson, K.W. 2009. Fluorescence In Situ Hybridization Confirms Clearance of Visible Helicobacter spp. Associated with Gastritis in Dogs and Cats. J. Vet. Intern. Med. 23: 16-23. doi: 10.1111/j.1939-1676.2008.0211.x

Joosten, M., Blaecher, C., Flahou, B., Ducatelle, R., Haesebrouck, F. \& Smet, A. 2013. Diversity in bacterium-host interactions within the species Helicobacter heilmannii sensu stricto. Vet. Res. 44: 65. doi:10.1186/1297-9716-44-65

Joosten, M., Lindén, S., Rossi, M., Chin Yen Tay, A., Skoog, E., Padra, M., Peters, F., Perkins, T., Vandamme, P., Van Nieuwerburgh, F., D’Herde, K., Van den Broeck, W., Flahou, B., Deforce, D., Ducatelle, R., Marshall, B., Haesebrouck, F. \& Smet, A. 2016. Divergence between the Highly Virulent Zoonotic Pathogen Helicobacter heilmannii and Its Closest Relative, the Low-Virulence "Helicobacter ailurogastricus" sp. nov. Infect. Immun. 84: 293-306. doi:10.1128/IAI.01300-15

Mitchell, H.M., Rocha, G.A., Kaakoush, N.O., O’Rourke, J.L. \& Queiroz, D.M.M. 2014. The Family Helicobacteraceae. In: Rosenberger, E., DeLong, E.F., Lory, S., Stackebrandt, E. \& Thompson, F. (Eds.). The Prokaryotes. Springer Berlin Heidelberg, Berlin, Heidelberg, pp. 337-392. doi:10.1007/978-3-642-39044-9_275

Suárez-Esquivel, M., Alfaro-Alarcón, A., Guzmán-Verri C. \& Barquero-Calvo E. 2017. Analysis of the association between Helicobacter spp. burden and gastric lesions in dogs. Am J Vet Res. 78 (12). 1414-1420. doi: doi.org/10.2460/ajvr.78.12.1414 